\title{
Application of In Vitro Toxicology Approaches for the Evaluation of Next-Generation Nicotine Products
}

\author{
Marianna D. Gaca
}

$\mathbf{T}$ HE EMERGENCE AND CONSUMPTION of novel nicotine and tobacco products have grown significantly on a global scale, potentially offering a range of alternatives to conventional tobacco products. The e-cigarette category, in particular, has seen an increase in product innovation with many types of e-cigarettes being developed. At its core, an e-cigarette is an electronic handheld device that vaporizes an e-liquid. The e-liquid is typically a simple formulation made of nicotine, propylene glycol, and/or glycerine and flavorings, although non-nicotine and nonflavored variants are also commercially available. It has been estimated that there are more than 8000 commercially available flavors. There are three main types of e-cigarettes: "cig-a-likes" that look like cigarettes and may be rechargeable and/or disposable, "closed systems" in which the e-liquid is contained in a cartridge, and "open tank systems" that have refillable liquid tanks.

Although the health risks of e-cigarette use have not yet been well studied, two leading U.K. institutions, the government's Public Health England and the Royal College of Physicians, have recently reviewed the evidence available and concluded that these products could be as much as $95 \%$ safer than combustible cigarettes. ${ }^{1,2}$ Longer term health effects are not definitively known, and further studies measuring the longer term effects of these products are required. The U.S. Food and Drug Administration's Center for Tobacco Products and the European Union Tobacco Products Directive have made recommendations and issued guidance documents outlining the criteria for assessing these novel products. As part of these proposed frameworks, there is a substantial requirement for nonclinical testing that will undoubtedly provide the support for the assessment of these novel consumer products and contribute significantly to the knowledge base. There is, therefore, a great opportunity to harness nextgeneration in vitro toxicity testing approaches, such as those outlined in the National Research Council's 2007 report "Toxicity Testing in the 21 st Century," and draw- ing from already well-established experts, including scientists in academia, industrial and government sectors, non-governmental organizations, animal welfare groups, and developers of test systems to assess this rapidly evolving category.

In this special issue of Applied In Vitro Toxicology, a number of these approaches are explored.

The roundtable discussion explores the challenges and opportunities in harnessing 21 st century in vitro toxicology testing methods for the assessment of e-cigarettes-how can these new methods be used to rapidly screen products and e-liquids, should and how can these nonregulated methods be accepted for product safety assessment, how can lessons be taken from many stakeholder groups to ensure there is standardization of approaches. The panel members represented a wide range of viewpoints, including experts from test method developers, manufacturers, regulatory science, and in vitro validation experts who are leaders in the development and application of in vitro assays.

A Commentary and a Letter to the Editor outline the considerations for the regulatory acceptance of in vitro approaches, and how the most efficient way to optimize and standardize approaches will require cooperation across a broad stakeholder group, including the regulatory community, academia, industry sectors, and manufacturers of test methods. Ten original articles cover the broad field of in vitro applications, ranging from dosimetry considerations, to harnessing analytical techniques and the simplicity of simple lung cell systems to rapidly screen e-liquids, to more elaborate three-dimensional tissue models, complex aerosol delivery systems, and systems biology approaches to unravel the mechanistic pathways associated with biological responses to aerosols. In vitro respiratory and ex vivo/in vitro cardiovascular systems are also described, outlining potential assays and physiologically relevant end points to evaluate novel products. Finally, with the application of adverse outcome pathways (AOPs) as a framework

British American Tobacco R\&D Centre, Southampton, United Kingdom.

(C) Marianna D. Gaca, 2017; Published by Mary Ann Liebert, Inc. This Open Access article is distributed under the terms of the Creative Commons Attribution Noncommercial License (http://creativecommons.org/licenses/by-nc/4.0/), which permits any noncommercial use, distribution, and reproduction in any medium, provided the original author and the source are credited. 
for risk assessment, this special issue presents two novel AOPs: decreased lung function and the development of hypertension as tools to help integrate in vitro findings and clinical data across key events.

Never before has there been such an exciting yet challenging time for the application of in vitro tools in supporting consumer product safety assessment. There is a wealth of new technologies available to enable faster, more accurate, and more physiologically relevant testing. In the case of e-cigarette toxicity testing, many considerations are required, ranging from the assessment of flavors and ingredients to the appropriate delivery of aerosols to test systems, to assessment of dose and extrapolation to consumer use, to the relevance of the in vitro test system to be used, and finally to the standardization of methods. These opportunities provide a rich environment for collaboration across various stakeholder groups, collectively working to develop and apply the most relevant in vitro tools for novel nicotine and tobacco product testing.

\section{Author Disclosure Statement}

This work was fully funded by British American Tobacco (Investments) Ltd. and Dr. Gaca is a full time employee of British American Tobacco (Investments) Ltd.

\section{References}

1. Public Health England. E-cigarettes: An Evidence Update. London: Public Health England; 2015.

2. Royal College of Physicians. Nicotine without Smoke: Tobacco Harm Reduction. London: RCP; 2016.

Address correspondence to: Marianna D. Gaca, PhD British American Tobacco $R \& D$ Centre Regents Park Road Southampton SO15 8TL United Kingdom

E-mail: marianna_gaca@bat.com 